\title{
Conductance of Alkanediisothiocyanates: Effect of Headgroup-Electrode Contacts
}

\author{
Ming-Dung Fu, ${ }^{\dagger}$ I-Wen Peter Chen, ${ }^{\dagger}$ Hao-Cheng Lu, ${ }^{\ddagger}$ Chun-Ting Kuo, ${ }^{\ddagger}$ \\ Wei-Hsiang Tseng, ${ }^{\dagger}$ and Chun-hsien Chen $*, *$ \\ Department of Chemistry, National Tsing Hua University, Hsinchu, Taiwan 30013, and Department of \\ Chemistry, National Taiwan University, Taipei, Taiwan 10617
}

Received: January 26, 2007; In Final Form: May 24, 2007

\begin{abstract}
The realization of molecular electronics requires comprehension of single-molecule $I-V$ characteristics. Aside from the electron-transport properties of the molecular framework, the molecule-electrode binding contributes significantly to the contact resistance, $R_{n=0}$, and thus to the values of single-molecule resistance. Isothiocyanate (-NCS), a versatile ligand for organometallics, can bind to a metal substrate to complete a metal-moleculemetal configuration for external measurements. Isothiocyanate has the advantage of being a $\pi$-conjugated moiety that presumably exhibits a relatively smaller impedance than the commonly used methylene thiol headgroup $\left(-\mathrm{CH}_{2} \mathrm{SH}\right)$ in many molecular wires. For example, this study shows that the single-molecule conductance of $n$-butanediisothiocyanate is an order of magnitude better than that of $n$-octanedithiol even though they both contain 10 atoms counted from sulfur to sulfur. For a homologous series of molecules, $R_{n=0}$ can be extrapolated from the intercept of the resistance obtained by the repeated formation of molecular junctions using scanning tunneling microscopy. To isolate the contact effect of the $-\mathrm{NCS}-\mathrm{Au}$ electrode from other factors, alkanediisothiocyanates were studied because the large HOMO-LUMO gap of alkyl chains is not sensitive to the number of methylene units. The results show two sets of $R_{n=0}$ values, with the smaller set being $128 \mathrm{k} \Omega$, about $1 / 12$ the other value. A detailed examination of the results suggests that the preferential adsorption site for isothiocyanate on gold is the atop site rather than the 3-fold-hollow sites of thiol on gold.
\end{abstract}

\section{Introduction}

The development of surface functionalization via selfassembled monolayers (SAMs) consistently gives rise to groundbreaking scientific findings and innovative applications. ${ }^{1-5}$ Such tailored molecules consist of a so-called headgroup that selectively exhibits affinity to certain substrates..$^{5-7}$ The most extensively studied combination of headgroups and surfaces is SAMs of organosulfurs on gold. The thiol headgroup is most commonly tethered to the functionality of interest through a methylene chain. In comparison to the saturated and somewhat flexible alkyl backbone of the thiols, isothiocyanate $(\mathrm{S}=\mathrm{C}=\mathrm{N})$ offers an electron-rich and relatively rigid linkage that has been shown to anchor organometallics, ${ }^{8-10}$ porphyrins, ${ }^{11,12}$ and other moieties ${ }^{13-15}$ onto the surfaces of coinage metals. From the aspect of future applications in molecular electronics, the $\pi$-conjugated characteristics of $\mathrm{SCN}^{-}$presumably allow throughbond electron transport more efficiently than the saturated methylene units.

Metal-molecule-metal (MMM) junctions offer a primitive configuration for measuring single-molecule conductance. Studies of scanning tunneling microscopy (STM) break junctions, ${ }^{16-18}$ conductive atomic force microscopy (AFM),${ }^{19-21}$ and theoretical simulations ${ }^{22,23}$ have shown that the molecule-metal contact has a substantial impact on the electric current passing through the molecule. Despite the aforementioned usefulness of $\mathrm{SCN}^{-}$, the headgroup-substrate interactions for SCN-terminated molecules have not been examined. Presented herein is an STM

* To whom correspondence should be addressed. E-mail: chhchen@ ntu.edu.tw.

$\dagger$ National Tsing Hua University.

$\doteqdot$ National Taiwan University. break-junction $^{16-18}$ study of alkanediisothiocyanates [SCN$\left(\mathrm{CH}_{2}\right)_{n} \mathrm{NCS}, n=4,6$, and 8] whose resistance values lead to an $R_{0}$ value determined by a linear extrapolation to the intercept against the number of methylene units, $n$. To simplify the effect of $-\mathrm{NCS}-\mathrm{Au}$ contact on molecule resistance, the poorly conductive saturated polymethylene chain was studied because its large HOMO-LUMO gap is not affected by the systematic change of $n$. The MMM junction was created by repeatedly pushing a gold tip into a gold surface submerged in toluene containing alkanediisothiocyanates and then retracting the tip away from the surface. The gold tip fuses with the substrate during the pushing step. While the tip is being pulled away, the merged tip-substrate joint breaks, and one or more molecules might bridge the junction. ${ }^{16-18,24-26}$ In this article, we discuss the $I-V$ characteristics of the metal-insulator-metal system, including the single-molecule resistance of alkanediisothiocyanates, the $-\mathrm{NCS}-\mathrm{Au}$ contact resistance, the tunneling decay constant per methylene unit, and the preferential binding site of isothiocyanate on gold.

\section{Experimental Section}

The experimental procedures and data treatment for STM break-junction studies were documented in detail by the groups of Tao and Lindsay. ${ }^{16-18}$ The experiments reported herein were carried with a NanoScope IIIa controller (NanoScope IIIa, Veeco, Santa Barbara, CA). The instrument was first operated in imaging mode with a tunneling current of $1-5 \mathrm{nA}$. When the images exhibited gold terraces, indicative of smooth surface and a sharp tip, the imaging mode was switched to STS plot $I(s)$ mode (scanning tunneling spectroscopy). The gold tip was brought (at a rate of $4.2-5.6 \mathrm{~nm} / \mathrm{s}, 0.93 \mathrm{~Hz}$ ) into and out of contact with a gold substrate in toluene (TEDIA). While current- 


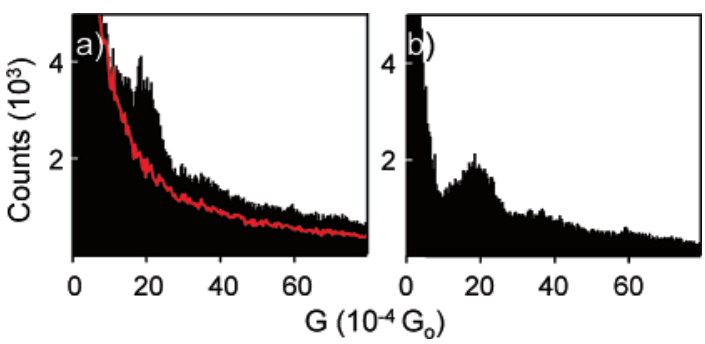

Figure 1. Conductance histograms constructed (a) from 1000 traces without preselection and (b) from 274 traces preselected by excluding noisy traces and those with simple tunneling. The experiments were carried out in toluene containing $1 \mathrm{mM} \mathrm{SCN}-\left(\mathrm{CH}_{2}\right)_{4}-\mathrm{NCS}$.

to-tip stretching profiles, $I(s)$, were being acquired, the feedback loop was off except at the beginning point of the cycle. Regardless of whether the solution contained the molecules of interest, the $I(s)$ traces exhibited a stepwise profile whose steps showed multiples of $G_{0}$, the conductance quantum being exchanged when the cross section of a metallic contact was that of only a single gold atom $\left[G_{0}=2 e^{2} / h, \sim(12.9 \mathrm{k} \Omega)^{-1}\right] .{ }^{27}$

The concentration of $n$-alkanediisothiocyanates or $n$-alkanedithiols in toluene was $1-20 \mathrm{mM}$. Upon repeated formation of the tip-substrate gap, the isothiocyanate or thiol headgroups at the termini of the polymethylene chains might bind to the gold electrodes and complete a molecular junction. The frequency for collecting the stepwise traces was about $27 \%$, slightly smaller than the $\sim 33 \%$ reported $^{18}$ for dithiol headgroups, presumably because of the stronger thiol-gold adsorption. The stepwise $I(s)$ traces were obtained at a fixed bias voltage, and the magnitudes were orders of magnitude smaller than $G_{0}$. To facilitate the determination of peak positions, the preparation of the conductance histogram excluded $I(s)$ traces with simple tunneling decay that were acquired when no molecule bridged between the electrodes. Tao et al. demonstrated that incorporating all of the traces in the histogram does not change the peak positions for single-molecule conductance of alkanedithiols, alkanediamines, and alkanedicarboxylic acids. ${ }^{16-18}$ Our finding for alkanediisothiocyanates agrees well with Tao et al.'s. For example, panel a of Figure 1 was plotted from 1000 traces without preselection and shows a peak at $20 \times 10^{-4} G_{0}$. The red curve indicates the contribution from 726 traces with either plain tunneling decay or uncharacterizable noise. The remaining 274 traces were redrawn and transferred panel b, where the peak position is almost identical to that in panel a.

The $I(s)$ traces were recorded with a built-in NanoScope program and exported as ASCII files. Each file had 5120 data points. Each of the 5120 data points for a given stepwise trace was converted to conductance and represented a value on the histogram ( $x$ axis), and over 2000 individual $I(s)$ traces were included for each molecule (vide infra, Figure 2), yielding a total sum of data points on the histogram of ca. $10^{7}$. The histogram indicates qualitatively how the conductance values were distributed, and only the range exhibiting peaks is shown. All of the histograms shown in this article were plotted by using 200 bins for the range presented. ${ }^{28}$ A Gaussian function was used to fit the histograms (Origin, version 6.0, Microcal Software, Inc.). The peak position and standard deviation of the Gaussian curve were used to find the single-molecule conductance and the error bars, respectively, in the subsequent figures.

All chemicals were reagent grade. Substrates were 100-nmthick gold films thermally evaporated onto glass slides with a 5-nm Cr underlayer (99.99\%, Super Conductor Materials, Inc., Suffern, NY) to enhance adhesion. The glass slides were precleaned with piranha solution, a $1: 3(\mathrm{v} / \mathrm{v})$ mixture of $30 \%$ $\mathrm{H}_{2} \mathrm{O}_{2}$ and concentrated $\mathrm{H}_{2} \mathrm{SO}_{4}$. Caution: This solution reacts violently with organics and should be handled with great care. The pressure in the bell-jar evaporator (Auto 306, Edwards High Vacuum International, West Sussex, U.K.) was nominally $2 \times$ $10^{-6}$ Torr. The $n$-alkanediisothiocyanates $\left[\mathrm{SCN}\left(\mathrm{CH}_{2}\right)_{n} \mathrm{NCS}, n\right.$ $=4$ from Alfa Aesar and $n=6,8$ from Oakwood Products] were used as received.

\section{Results and Discussion}

I(s) Traces and Conductance Histograms. Figure 2 shows the results of STM break-junction studies for alkanediisothiocyanates with 4,6 , and 8 methylene units. The upper panels of Figure 2 display typical $I(s)$ traces of electric current acquired when the STM tip was pulled away from the substrate and the bias voltage, $V_{\text {bias, }}$, between the tip and the substrate was held constant. The $I(s)$ traces are presented with arbitrary $x$-axis offsets. The abrupt drop associated with the tip stretching suggests a decrease in the number of molecules bridging the tip - substrate gap. The current values are scaled by $V_{\text {bias, }}$, and their quotient renders the conductance $G$ of single molecules when $V_{\text {bias }}$ is small. To be consistent with literature reports, ${ }^{16,17,27,29}$ the unit of the ordinate is $G_{0}$. Controlled experiments in blank toluene resulted in traces with simple exponential tunneling decay, confirming that the staircase waveforms arose from the alkanediisothiocyanates. The insets in the upper panels of Figure 2 reveal low-conductance (LC) steps with values approximately $1 / 10$ those of the high-conductive (HC) steps. The presence of multiple conductance values is ascribed to the different anchoring geometries on the substrate and has been reported for alkanedithiols, ${ }^{18,30}$ alkanediamines, ${ }^{18}$ and alkanedicarboxylic acids ${ }^{18}$ on gold where the conductance ratios of $\mathrm{HC}$ to $\mathrm{LC}$ values are about 4-5, 15, and 10, respectively.

The lengths of step terraces correspond to the durations or probabilities of alkanediisothiocyanates being sandwiched in the MMM junction. The histograms in the lower panels are summarized from more than $2000 I(s)$ traces and are plotted against $G_{0}$. The local maxima of occurrences are distributed at integer multiple of the fundamental values, suggesting that the number of molecules in the junctions is one, two, etc. ${ }^{16-18}$ The insets show histograms of conductance in the LC region. It is noteworthy that the histogram for octanediisothiocyanate exhibits a conductance peak in the LC region even when the extension of the LC steps is superimposed and narrowed by the tunneling background.

Comparison of SCN and Thiol Headgroups. As a control in this work, we also carried out experiments on the extensively studied alkanedithiols whose conductance results are consistent with literature reports $16,17,25,34$ and can be the platform for calibrating our measurements of alkanediisothiocyanates. Table 1 summarizes the single-molecule conductance values of two $\alpha, \omega$-alkanes for better comparison.

To illustrate the effect of the delocalized $\pi$ electrons at SCN on the single-molecule resistance, Figure 3shows a plot of the values from Table 1 against the total number of atoms in the molecules. For pairs of molecules with the same number of atoms, namely, $\mathrm{Au}-\mathrm{SCN}\left(\mathrm{CH}_{2}\right)_{4} \mathrm{NCS}-\mathrm{Au}$ versus $\mathrm{Au}-\mathrm{S}\left(\mathrm{CH}_{2}\right)_{8} \mathrm{~S}-$ $\mathrm{Au}$ and $\mathrm{Au}-\mathrm{SCN}\left(\mathrm{CH}_{2}\right)_{6} \mathrm{NCS}-\mathrm{Au}$ versus $\mathrm{Au}-\mathrm{S}\left(\mathrm{CH}_{2}\right)_{10} \mathrm{~S}-\mathrm{Au}$, the $\mathrm{HC}$ region of the alkanediisothiocyanates (solid squares) is 

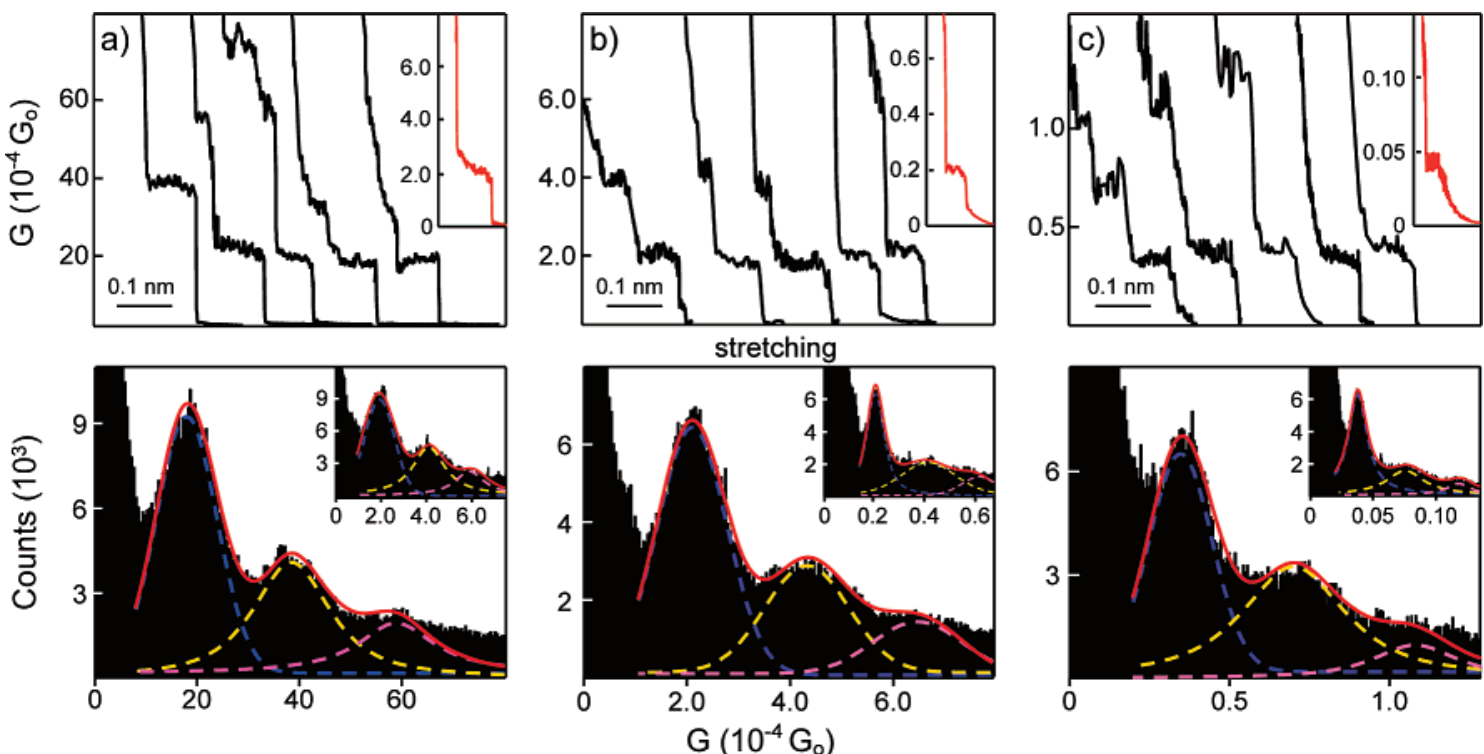

Figure 2. Single-molecule conductance measurements obtained by STM break-junction studies of (a) butanediisothiocyanate, (b) hexanediisothiocyanate, and (c) octanediisothiocyanate. Upper panels: Typical current-distance traces acquired upon stretching of the molecular junctions. The vertical axes represent conductance values, converted from the measured currents by dividing by the corresponding $V_{\text {bias }}$ and $G_{0}$ values. The conductance of the traces decreases in quantized steps because of the formation of a stable molecular junction between the gold STM tip and the gold substrate. ${ }^{16-18,24-26,30-33}$ Lower panels: Conductance histograms plotted from more than $2000 I(s)$ traces. The experiments were carried out in toluene and under a $\mathrm{N}_{2}(\mathrm{~g})$-purged environment. In the absence of alkanediisothiocyanates, no such steps or peaks were observed within the same conductance range. Insets: $I(s)$ traces and histograms for low-conductance sets whose conductance values are an order of magnitude smaller than those of the high-conductance sets. Peak positions were determined using Gaussian fits to the histograms profiles. The blue lines are the sums of the Gaussians. The standard deviations estimated from the Gaussian fittings were used as the error bars in the subsequent figures.

TABLE 1: Summary of Single-Molecule Conductance and Resistance Values for Alkanediisothiocyanates and Alkanedithiols

\begin{tabular}{|c|c|c|c|c|c|c|c|c|}
\hline \multirow[b]{3}{*}{$n$} & \multicolumn{4}{|c|}{$\mathrm{Au}-\mathrm{SCN}-\left(\mathrm{CH}_{2}\right)_{n}-\mathrm{NCS}-\mathrm{Au}$ junctions } & \multicolumn{4}{|c|}{$\mathrm{Au}-\mathrm{S}-\left(\mathrm{CH}_{2}\right)_{n}-\mathrm{S}-\mathrm{Au}$ junctions } \\
\hline & \multicolumn{2}{|c|}{$\mathrm{HC}^{a}$} & \multicolumn{2}{|c|}{$\mathrm{LC}^{a}$} & \multicolumn{2}{|c|}{$\mathrm{HC}^{a}$} & \multicolumn{2}{|c|}{$\mathrm{LC}^{a}$} \\
\hline & $\overline{G\left(\times 10^{-4} G_{0}\right)^{b}}$ & $R(\mathrm{M} \Omega)^{b}$ & $\overline{G\left(\times 10^{-4} G_{0}\right)}$ & $R(\mathrm{M} \Omega)$ & $\overline{G\left(\times 10^{-4} G_{0}\right)}$ & $R(\mathrm{M} \Omega)$ & $\overline{G\left(\times 10^{-4} G_{0}\right)}$ & $R(\mathrm{M} \Omega)$ \\
\hline 4 & $19 \pm 4$ & $6.7 \pm 1.4$ & $2.0 \pm 0.4$ & $65 \pm 14$ & - & - & - & - \\
\hline 6 & $2.0 \pm 0.4$ & $66 \pm 14$ & $0.20 \pm 0.04$ & $629 \pm 134$ & $12 \pm 2$ & $10.7 \pm 2.0$ & $2.2 \pm 0.4$ & $59 \pm 12$ \\
\hline 8 & $0.34 \pm 0.07$ & $379 \pm 80$ & $0.043 \pm 0.009$ & $3012 \pm 653$ & $2.6 \pm 0.4$ & $49.1 \pm 7.3$ & $0.59 \pm 0.06$ & $218 \pm 21$ \\
\hline 10 & & - & - & - & $0.22 \pm 0.04$ & $600 \pm 120$ & $0.043 \pm 0.009$ & $3000 \pm 600$ \\
\hline
\end{tabular}

${ }^{a} \mathrm{HC}$ and LC denote high- and low-conductance datasets, respectively. ${ }^{b}$ The values of $G$ and $R$ are interconvertible through $G_{0}=(12.9 \mathrm{k} \Omega)^{-1}$. The standard deviations were obtained from Gaussian fittings.

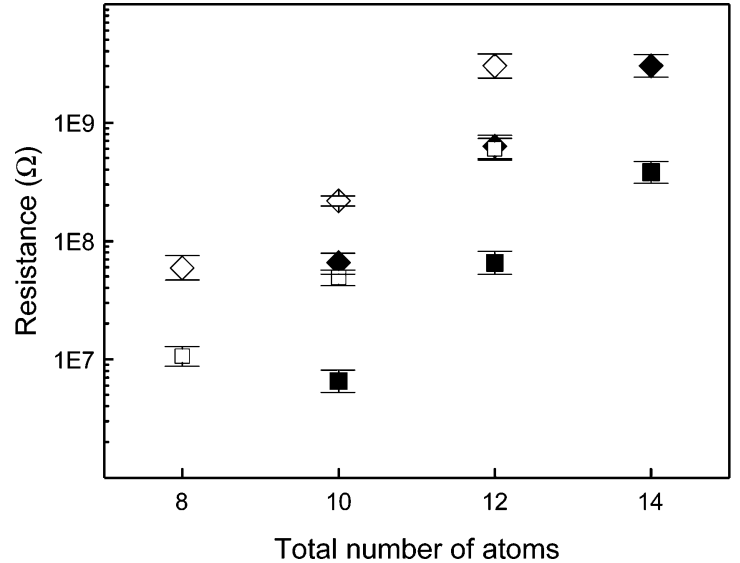

Figure 3. Semilogarithmic plots of single-molecule resistance versus total number of atoms in the molecules, counted from sulfur to sulfur. Alkanediisothiocyanates: $\mathbf{\square}, \mathrm{HC} ; \diamond$, LC. Alkanedithiols: $\square, \mathrm{HC} ; \diamond$, LC.

an order of magnitude more conductive than the $\mathrm{HC}$ region of the alkanedithiols (open squares). The LC values of alkanediisothiocyanates (solid diamonds) are either similar to the $\mathrm{HC}$ values of alkanedithiols or about 4 times more conductive than the corresponding LC regions of the alkanedithiols (open diamonds).
The electronic coupling (or tunneling decay) constant, $\beta_{n}$, for a homologous series is extracted from the relation $R \propto$ $\exp \left(-\beta_{n} n\right)$ or $\exp (-\beta L)$, where $n$ and $L$ are the number of methylene units and the molecular length (in angstroms), respectively. From the control experiments on alkanedithiols reported in Table 1 , a $\beta_{n}$ value of ca. 1.0 is derived (Table 2), in good agreement with the consensus values reported in the literature. ${ }^{16,35,36}$ For alkanediisothiocyanates, the semilogarithmic plots of $R$ with respect to $n$ are presented in Figure 4a. The straight lines indicate an exponential increase in the molecular resistance with increasing molecular chain length, suggesting a coherent and nonresonant tunneling. The slopes of the leastsquares lines give $\beta_{n}$ values of ca. 1.0 per $\mathrm{CH}_{2}$ group for both $\mathrm{HC}$ and LC sets of alkanediisothiocyanates (see Table 2). The fact that the $\beta_{n}$ values are practically identical to those of alkanedithiols confirms that the discrepancy in their singlemolecule resistances should arise from the terminal groups. Panels a and b of Figure 4 show that extrapolation of the leastsquares fitting to the intercept gives respective $\mathrm{HC}$ and $\mathrm{LC}$ contact resistances, $R_{n=0}$, of $105 \mathrm{k} \Omega$ and $1.36 \mathrm{M} \Omega$ for the alkanediisothiocyanates and 22 and $130 \mathrm{k} \Omega$ for the alkanedithiols. Although the former have larger $R_{n=0}$ values, the contact consists of two NCS-Au units whose headgroup-electrode junctions, in fact, contain a total of four bonds that are longer than those of dithiols. 
TABLE 2: Summary of $\boldsymbol{\beta}_{n}, \boldsymbol{R}_{n=0}$, and Frequency of Observation for $\mathrm{HC}^{a}$ and $\mathrm{LC}^{a}$ Datasets for Alkanediisothiocyanates and Alkanedithiols

\begin{tabular}{llllll}
\hline & \multicolumn{2}{c}{$\begin{array}{c}\mathrm{Au}-\mathrm{SCN}-\left(\mathrm{CH}_{2}\right)_{n}- \\
\mathrm{NCS}-\mathrm{Au} \text { junctions }\end{array}$} & & \multicolumn{2}{c}{$\begin{array}{c}\mathrm{Au}-\mathrm{S}-\left(\mathrm{CH}_{2}\right)_{n}- \\
\mathrm{S}-\mathrm{Au} \text { junctions }\end{array}$} \\
\cline { 2 - 3 } \cline { 5 - 6 } & \multicolumn{1}{c}{$\mathrm{HC}$} & \multicolumn{1}{c}{$\mathrm{LC}$} & & $\mathrm{HC}$ & \multicolumn{1}{c}{$\mathrm{HC}$} \\
\hline$\beta_{n}\left(\right.$ per $\left.\mathrm{CH}_{2}\right)$ & $1.01 \pm 0.07$ & $0.96 \pm 0.07$ & & $1.01 \pm 0.14$ & $0.98 \pm 0.19$ \\
$\beta($ per $\AA$ ) & $0.80 \pm 0.06$ & $0.75 \pm 0.05$ & & $0.79 \pm 0.11$ & $0.77 \pm 0.15$ \\
$R_{n=0}(\mathrm{k} \Omega)^{b}$ & $128 \pm 59$ & $1560 \pm 720$ & & $22 \pm 25$ & $130 \pm 200$ \\
$G_{n=0}\left(G_{0}\right)^{b}$ & $0.10 \pm 0.05$ & $0.008 \pm 0.004$ & & $0.60 \pm 0.70$ & $0.10 \pm 0.15$ \\
probability & $34^{d}$ & $58^{d}$ & 63 & 37 \\
$(\%)^{c}$ & & & & &
\end{tabular}

${ }^{a} \mathrm{HC}$ and LC denote high- and low-conductance datasets, respectively. ${ }^{b} R_{n=0}$ and $G_{n=0}$ are, respectively, the contact resistance and contact conductance obtained from the intercepts of Figure 4. $G_{0}=$ $2 e^{2} / h \approx 77.4 \mu \mathrm{S}$ or $(12.9 \mathrm{k} \Omega)^{-1} .{ }^{c}$ Frequency of occurrence reported for $\mathrm{SCN}-\left(\mathrm{CH}_{2}\right)_{4}-\mathrm{NCS}$ and $\mathrm{HS}-\left(\mathrm{CH}_{2}\right)_{6}-\mathrm{SH}$ because of their larger current values, better-defined $I(s)$ traces, and thus more conclusive results than obatined for the other molecules. ${ }^{d}$ Approximately $8 \%$ of the traces exhibited both HC and LC steps; see text.

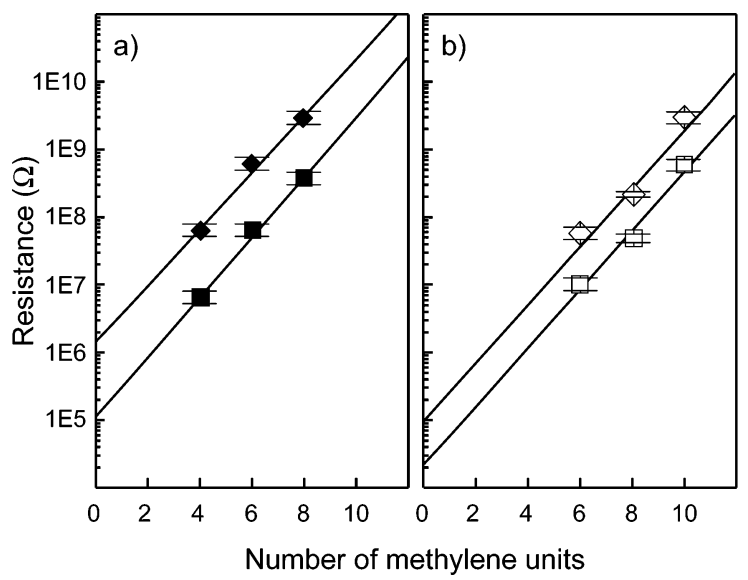

Figure 4. Semilogarithmic plot of single-molecule resistance versus number of methylene units for (a) $n$-alkanediisothiocyanates and (b) $n$-alkanedithiols. The slopes and $y$ intercepts of the best-fit lines yield the electronic coupling constant, $\beta_{n}$, and the contact resistance, $R_{n=0}$, respectively, for HC (squares) and LC (diamonds) groups.

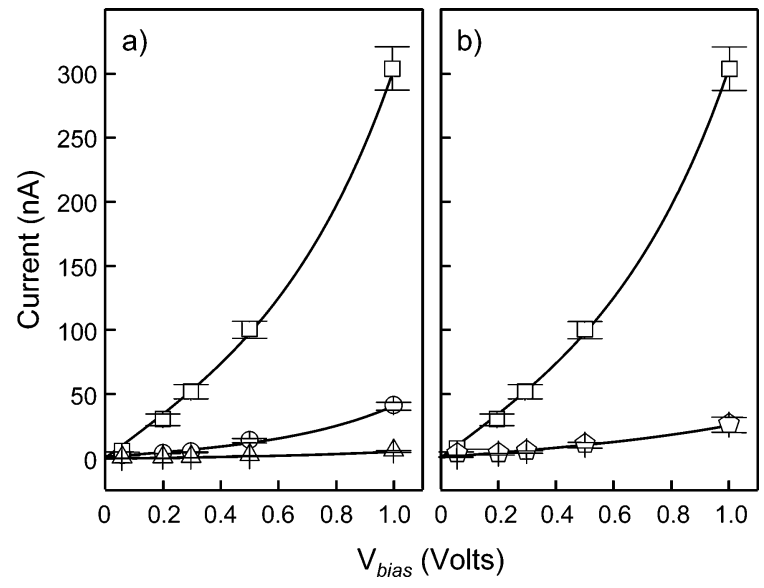

Figure 5. $I-V$ responses of alkanediisocyanates. (a) Positions of the fundamental peaks in the $\mathrm{HC}$ conductive histograms of butane(squares), hexane- (circles), and octanediisothiocyanates (triangles). (b) $I-V$ plots for butanediisothiocyanate of the HC (squares) and LC (pentagons) single-molecule conductance values. The curves are the best fits obtained using the Simmons formula ${ }^{37}$ (see text).

Current-Voltage Plots. Plotted in Figure 5 for the alkanediisothiocyanates are the current values of the fundamental peaks in the $I(s)$ histograms as a function of the corresponding bias voltages. The curves in Figure 5 are $I-V$ characteristics fitted by the Simmons model ${ }^{37}$

$$
\begin{aligned}
& I \propto e\left(\phi_{\mathrm{B}}-\right.\left.\frac{V_{\text {bias }}}{2}\right) \exp \left[-\frac{2(2 m)^{1 / 2} e^{1 / 2}}{\hbar} \alpha\left(\phi_{\mathrm{B}}-\frac{V_{\text {bias }}}{2}\right)^{1 / 2} L\right]- \\
& e\left(\phi_{\mathrm{B}}+\frac{V_{\text {bias }}}{2}\right) \exp \left[-\frac{2(2 m)^{1 / 2} e^{1 / 2}}{\hbar} \alpha\left(\phi_{\mathrm{B}}+\frac{V_{\text {bias }}}{2}\right)^{1 / 2} L\right]
\end{aligned}
$$

Derived from the Wentzel-Kramers-Brillouin approximation, ${ }^{38,39}$ this formulation indicates that, under an intermediatevoltage range of bias $V_{\text {bias }}$ (in volts), the current $I$ (in amperes) is a result of electron tunneling across a rectangular barrier of height $\phi_{\mathrm{B}}$ (in volts) between electrodes spaced by a thin insulating film with a thickness of $L$ (in meters). $m, e$, and $\hbar$ are the rest mass of an electron (in kilograms), the charge of an electron (in Coulombs), and Planck's constant divided by $2 \pi$ (in Joule seconds), respectively. To generalize the formulation for any shape of potential barrier, Simmons introduced the parameter $\alpha$, which, by itself, does not provide physical meaning about the structure of the barrier. ${ }^{37,40,41}$ Simmons' formula shows that, at a very small $V_{\text {bias }}$, the current is dependent on the barrier width $L$ as $i=\left(V_{\text {bias }} / \mathrm{R}\right) \mathrm{e}^{-\beta L}$ where $\beta$ (in $\left.\AA^{-1}\right)$ is a biasindependent decay constant.

$$
\beta=\frac{2(2 m)^{1 / 2} \mathrm{e}^{1 / 2}}{\hbar} \alpha \phi_{\mathrm{B}}^{1 / 2} \approx 1.03 \alpha \phi_{\mathrm{B}}^{1 / 2}
$$

For molecules having a larger value of $n$, the conducting current decreases exponentially, expressed by $i=\left(V_{\text {bias }} / R\right) \mathrm{e}^{-\beta_{n} n}$. For $I-V$ traces of the LC datasets, only butanediisothiocyanate was subjected to detailed examination because the riding of LC steps over the exponential decay curve of electron tunneling makes the data analysis more difficult for the longer molecules. Taking the insets of panels $b$ and $c$ of Figure 2 as examples, the LC currents at the fundamental steps are no longer significantly larger than the current of through-space tunneling for hexaneand octanediisothiocyanates.

By employing $L$ values of 1.12, 1.37, and $1.62 \mathrm{~nm}$ for butane-, hexane-, and octanediisothiocyanates, respectively, the best-fit results for $\alpha$ and $\phi_{\mathrm{B}}$ for the $\mathrm{HC}$ sets were found to be 0.61 $( \pm 0.01)$ and $1.36( \pm 0.03) \mathrm{eV}, 0.68( \pm 0.04)$ and $1.32( \pm 0.11)$ $\mathrm{eV}$, and $0.58( \pm 0.03)$ and $1.40( \pm 0.13) \mathrm{eV}$. The molecular dimensions were estimated according to MM2 calculations (molecular mechanics calculator 2, embedded in ChemBats3D). These parameters yielded values for the constants $\beta$ of 0.71 $( \pm 0.01), 0.78( \pm 0.12)$, and $0.72( \pm 0.14) \AA^{-1}$ for butane-, hexane-, and octanediisothiocyanates, respectively. The LC sets for butanediisothiocyanate resulted in values of $0.64( \pm 0.05)$, $1.36( \pm 0.18) \mathrm{eV}$, and $0.75( \pm 0.09) \AA^{-1}$ for $\alpha, \phi_{\mathrm{B}}$, and $\beta$, respectively. These best-fitt parameters are indistinguishable even though their current values are different by orders of magnitude. Both the $\phi_{\mathrm{B}}$ and $\beta$ are slightly smaller than those of alkanedithiols, ${ }^{41,42}$ because of the difference between the conjugated and saturated headgroups.

Discussion of SCN Binding Geometry. Measurements of STM break junctions have resolved multiple sets of singlemolecule conductance values for $\alpha, \omega$-alkanes of dithiols, ${ }^{17,18,32,33}$ diamines, ${ }^{18}$ and dicarboxylic acids. ${ }^{18}$ The populations of the HC $I(s)$ traces are reported to be about 2-3 times those of the LC sets. ${ }^{17,18}$ The origin of the $\mathrm{HC}$ and LC sets is attributed to the microstructures at the region of headgroup-electrode contact, which vary concomitantly with the repeatedly created molecular junctions. In the case of alkanedithiols, Lindsay, Tao, and coworkers ${ }^{17}$ generalized the HC and LC contacts to molecular 
termini sitting on atop and hollow sites of the gold substrate (denoted as atop-hollow) and on two atop sites (atop-atop), respectively, on the basis of the following observations: (1) Simultaneous measurements of force and single-molecule conductance values reveal the same breakdown force of $\sim 1.5$ $\mathrm{nN}$ for both the $\mathrm{HC}$ and $\mathrm{LC}$ traces. This value is the same as that required to break the $\mathrm{Au}-\mathrm{Au}$ bond, ${ }^{43}$ suggesting that the $\mathrm{S}-\mathrm{Au}$ bond is strong enough to pull the gold atoms from the 3-fold-hollow sites $\left(\mathrm{R}-\mathrm{S}-\mathrm{Au}_{3}\right)$ to atop sites $(\mathrm{R}-\mathrm{S}-\mathrm{Au})$. Therefore, when the molecular junction breaks, one of the terminal sulfur atoms resides on an atop site. (2) $I(s)$ traces with both $\mathrm{HC}$ and LC features, although rare, always exhibit an HC step first and subsequently an LC step. The lack of a current trace going from LC to $\mathrm{HC}$ agrees with the progression of junction breakage that $\mathrm{S}-\mathrm{Au}$ binding can be transformed from anchoring at a hollow site into an atop site, but not in the opposite direction. (3) According to probability considerations, the frequency of the atop-hollow configuration should be twice that of the atop-atop scheme.

Our control experiments on hexanedithiol confirm that the probability of obtaining $\mathrm{HC}$ traces is higher than that of obtaining LC traces (Table 2), consistent with the literature reports. ${ }^{17,18}$ For butanediisothiocyanate, the occurrences of HC and LC traces are about $34 \%$ and $58 \%$, respectively. The remaining $\sim 8 \%$ exhibit both $\mathrm{HC}$ and $\mathrm{LC}$ steps. $\mathrm{HC}$ steps in those traces always appear first, with an LC step following. The reverse sequence was not observed. The rationale offered by Lindsay, Tao, and co-workers ${ }^{17}$ that ascribes the HC and LC steps to atop-hollow and atop-atop contact geometries, respectively, also explains well the behavior of butanediisothiocyanate on gold, except that the HC traces have a lower occurrence frequency than the LC ones. This discrepancy is explained by the density function theory (DFT) modeling of $\mathrm{Xu}$ and Gewirth who found that $\mathrm{S}-\mathrm{Au}$ adsorption of isothiocyanate on the atop site is $0.364 \mathrm{eV}$ more stable than that on the hollow site of $\mathrm{Au}(111) .{ }^{44}$ Therefore, the distribution of the $\mathrm{HC}$ and LC traces is likely not dictated purely by random combination. The favorable adsorption energy at the atop sites renders a higher occurrence for the atop-atop configuration, which, however, has a smaller conductance because it has fewer $\mathrm{S}-\mathrm{Au}$ contacts.

\section{Conclusion}

Two conductance sets were found for alkanediisothiocyanates and were ascribed to their different $\mathrm{Au}-\mathrm{SCN}\left(\mathrm{CH}_{2}\right)_{n} \mathrm{NCS}-\mathrm{Au}$ binding geometries. The probability of acquiring the $\mathrm{HC}$ set is $34 \%$, smaller than the $58 \%$ probability of the LC set. This trend is opposite to the case of alkanedithiols, whose $\mathrm{HC}$ occurrence is 2-3 times that of LC. According to DFT modeling in which the energy for SCN adsorption on the atop sites is $0.364 \mathrm{eV}$ more stable than that on the hollow sites of a gold substrate, we propose that the HC and LC traces are obtained when the SCN resides on atop-hollow and atop-atop sites, respectively. Their tunneling decay constants of ca. 1.0 per $\mathrm{CH}_{2}$ are indistinguishable from those of alkanedithiols. The singlemolecule conductance valaues of alkanediisothiocyanates are an order of magnitude better than those of alkanedithiols with the same number of atoms between the terminal sulfur atoms. The $\mathrm{S}=\mathrm{C}=\mathrm{N}$ headgroup with delocalized $\pi$ electrons is responsible for the good electron-transport properties and, therefore, functions as a suitable ligand as well as a contact lead for organometallic wires.

Acknowledgment. The authors thank National Tsing Hua University and National Taiwan University for the strong research support. C.-h.C acknowledges the College of Science (NTU) for startup funding (95R0034-02). This work was supported by the National Science Council of R.O.C. through Grants NSC-95-2113-M-002-036 and NSC-95-2752-M-002001-PAE.

\section{References and Notes}

(1) Troisi, A.; Ratner, M. A. Small 2006, 2, 172-181.

(2) Delamarche, E.; Juncker, D.; Schmid, H. Adv. Mater. 2005, 17 2911-2933.

(3) Gates, B. D.; Xu, Q.; Stewart, M.; Ryan, D.; Willson, C. G.; Whitesides, G. M. Chem. Rev. 2005, 105, 1171-1196.

(4) Senaratne, W.; Andruzzi, L.; Ober, C. K. Biomacromolecules 2005, 6, 2427-2448.

(5) Love, J. C.; Estroff, L. A.; Kriebel, J. K.; Nuzzo, R. G.; Whitesides, G. M. Chem. Rev. 2005, 105, 1103-1169.

(6) Ulman, A. Chem. Rev. 1996, 96, 1533-1554.

(7) Schwartz, D. K. Annu. Rev. Phys. Chem. 2001, 52, 107-137.

(8) Lin, S.-Y.; Chen, I-W. P.; Chen, C.-h.; Hsieh, M.-H.; Yeh, C.-Y.; Lin, T.-W.; Chen, Y.-H.; Peng, S.-M. J. Phys. Chem. B 2004, 108, 959964.

(9) Tadayyoni, M. A.; Farquharson, S.; Li, T. T.-T.; Weaver, M. J. J. Phys. Chem. 1984, 88, 4701-4706.

(10) Chen, I-W. P.; Fu, M.-D.; Tseng, W.-H.; Yu, J.-Y.; Wu, S.-H.; Ku, C.-J.; Chen, C.-h.; Peng, S.-M. Angew. Chem., Int. Ed. 2006, 45, 58145818 .

(11) Han, W.; Li, S.; Lindsay, S. M.; Gust, D.; Moore, T. A.; Moore, A. L. Langmuir 1996, 12, 5742-5744.

(12) Han, W.; Durantini, E. N.; Moore, T. A.; Moore, A. L.; Gust, D.; Rez, P.; Leatherman, G.; Seely, G. R.; Tao, N.; Lindsay, S. M. J. Phys. Chem. B 1997, 101, 10719-10725.

(13) Singh, M. M.; Rastogi, R. B.; Upadhyay, B. N.; Yadav, M. Mater. Chem. Phys. 2003, 80, 283-293.

(14) Pranger, L.; Goldstein, A.; Tannenbaum, R. Langmuir 2005, 21, 5396-5404.

(15) Pettinger, B.; Ren, B.; Picardi, G.; Schuster, R.; Ertl, G. Phys. Rev Lett. 2004, 92, 096101.

(16) Xu, B.; Tao, N. J. Science 2003, 301, 1221-1223.

(17) Li, X.; He, J.; Hihath, J.; Xu, B.; Lindsay, S. M.; Tao, N. J. Am Chem. Soc. 2006, 128, 2135-2141.

(18) Chen, F.; Li, X.; Hihath, J.; Huang, Z.; Tao, N. J. Am. Chem. Soc. 2006, 128, 15874-15881.

(19) Beebe, J. M.; Engelkes, V. B.; Miller, L. L.; Frisbie, C. D. J. Am. Chem. Soc. 2002, 124, 11268-11269.

(20) Engelkes, V. B.; Beebe, J. M.; Frisbie, C. D. J. Am. Chem. Soc. 2004, 126, 14287-14296.

(21) Kim, B. S.; Beebe, J. M.; Jun, Y.; Zhu, X.-Y.; Frisbie, C. D. J. Am. Chem. Soc. 2006, 128, 4970-4971.

(22) Seminario, J. M.; De La Cruz, C. E.; Derosa, P. A. J. Am. Chem. Soc. 2001, 123, 5616-5617.

(23) Basch, H.; Cohen, R.; Ratner, M. A. Nano Lett. 2005, 5, $1668-$ 1675 .

(24) Venkataraman, L.; Klare, J. E.; Nuckolls, C.; Hybertsen, M. S.; Steigerwald, M. L. Nature 2006, 442, 904-907.

(25) Ulrich, J.; Esrail, D.; Pontius, W.; Venkataraman, L.; Millar, D.; Doerrer, L. H. J. Phys. Chem. B 2006, 110, 2462-2466.

(26) Venkataraman, L.; Klare, J. E.; Tam, I. W.; Nuckolls, C.; Hybertsen, M. S.; Steigerwald, M. L. Nano Lett. 2006, 6, 458-462.

(27) Ohnishi, H.; Kondo, Y.; Takayanagi, K. Nature 1998, 395, 780783.

(28) The peak height was dependent of the bin size or the number of bins in the presented conductance range. The number of counts for the peak of single-molecule conductance (that is, the area under the peak) was $\sim 10^{4}$, in good agreement with the $\sim 150$ data points per step for $\sim 0.1-\mathrm{nm}$ steps at the operating conditions employed $(4.2-5.6 \mathrm{~nm} / \mathrm{s}, 0.93 \mathrm{~Hz}, 2000$ traces). The count numbers appeared smaller than what was estimated because there were two types of traces, high-conductance and low-conductance, whose probabilities were found roughly one-third and two-thirds, respectively, of the stepwise traces (see Table 2).

(29) Yanson, A. I.; Rubio Bollinger, G.; van den Brom, H. E.; Agrait, N.; van Ruitenbeek, J. M. Nature 1998, 395, 783-785.

(30) Ishizuka, K.; Suzuki, M.; Fujii, S.; Takayama, Y.; Sato, F.; Fujihira, M. Jpn. J. Appl. Phys. 2006, 45, 2037-2040.

(31) Chen, F.; Nuckolls, C.; Lindsay, S. Chem. Phys. 2006, 324, 236243.

(32) Suzuki, M.; Fujii, S.; Fujihira, M. Jpn. J. Appl. Phys. 2006, 45, 2041-2044.

(33) Fujihira, M.; Suzuki, M.; Fujii, S.; Nishikawa, A. Phys. Chem. Chem. Phys. 2006, 8, 3876-3884. 
(34) Salomon, A.; Cahen, D.; Lindsay, S.; Tomfohr, J.; Engelkes, V. B.; Frisbie, C. D. Adv. Mater. 2003, 15, 1881-1890.

(35) Lee, T.; Wang, W.; Klemic, J. F.; Zhang, J. J.; Su, J.; Reed, M. A. J. Phys. Chem. B 2004, 108, 8742-8750.

(36) Adams, D. M.; Brus, L.; Chidsey, C. E. D.; Creager, S.; Creutz, C.; Kagan, C. R.; Kamat, P. V.; Lieberman, M.; Lindsay, S.; Marcus, R. A.; Metzger, R. M.; Michel-Beyerle, M. E.; Miller, J. R.; Newton, M. D.; Rolison, D. R.; Sankey, O.; Schanze, K. S.; Yardley, J.; Zhu, X. J. Phys. Chem. B 2003, 107, 6668-6697.

(37) Simmons, J. G. J. Appl. Phys. 1963, 34, 1793-1803.

(38) Messiah, A. Classical Approximation and the WKB Method. Quantum Mechanics; North-Holland Publishers: Amsterdam, 1964; Chapter 6 , pp 214-242.
(39) Davydov, A. S. The Connection Between Quantum Mechanics and Classical Mechanics. Quantum Mechanics, 2nd ed.; Pergamon Press: Oxford, U.K., 1976; Chapter 3, pp 71-86.

(40) Holmlin, R. E.; Haag, R.; Chabinyc, M. L.; Ismagilov, R. F.; Cohen, A. E.; Terfort, A.; Rampi, M. A.; Whitesides, G. M. J. Am. Chem. Soc. 2001, 123, 5075-5085

(41) Wang, W.; Lee, T.; Reed, M. A. Phys. Rev. B 2003, 68, 035416

(42) Wang, W.; Lee, T.; Reed, M. A. Proc. IEEE 2005, 93, 1-11.

(43) Xu, B.; Xiao, X.; Tao, N. J. J. Am. Chem. Soc. 2003, 125, 1616416165.

(44) Li, X.; Gewirth, A. A. J. Am. Chem. Soc. 2003, 125, 1167411683. 